\title{
Analytical method to estimate resin cement diffusion into dentin
}

Larissa Cristina de Oliveira Ferraz

Adriana Lemos Mori Ubaldini

Bruna Medeiros Bertol de Oliveira

Antonio Medina Neto

Fracielle Sato

Mauro Luciano Baesso

Renata Corrêa Pascotto 


\title{
Analytical method to estimate resin cement diffusion into dentin
}

\author{
Larissa Cristina de Oliveira Ferraz, ${ }^{a}$ Adriana Lemos Mori Ubaldini, ${ }^{a}$ Bruna Medeiros Bertol de Oliveira, ${ }^{a}$ \\ Antonio Medina Neto, ${ }^{\mathrm{b}}$ Fracielle Sato, ${ }^{\mathrm{b}}$ Mauro Luciano Baesso, ${ }^{\mathrm{b}}$ and Renata Corrêa Pascotto ${ }^{\mathrm{a}, *}$ \\ aState University of Maringá, Department of Dentistry, Av. Mandacaru 1550, 87080-0009, Maringá, Paraná, Brazil \\ ${ }^{b}$ State University of Maringá, Department of Physics, Av. Colombo 5790, 87020-900, Maringá, Paraná, Brazil
}

\begin{abstract}
This study analyzed the diffusion of two resin luting agents (resin cements) into dentin, with the aim of presenting an analytical method for estimating the thickness of the diffusion zone. Class $\mathrm{V}$ cavities were prepared in the buccal and lingual surfaces of molars $(n=9)$. Indirect composite inlays were luted into the cavities with either a self-adhesive or a self-etch resin cement. The teeth were sectioned bucco-lingually and the cementdentin interface was analyzed by using micro-Raman spectroscopy (MRS) and scanning electron microscopy. Evolution of peak intensities of the Raman bands, collected from the functional groups corresponding to the resin monomer $\left(\mathrm{C}-\mathrm{O}-\mathrm{C}, 1113 \mathrm{~cm}^{-1}\right)$ present in the cements, and the mineral content $\left(\mathrm{P}-\mathrm{O}, 961 \mathrm{~cm}^{-1}\right)$ in dentin were sigmoid shaped functions. A Boltzmann function (BF) was then fitted to the peaks encountered at $1113 \mathrm{~cm}^{-1}$ to estimate the resin cement diffusion into dentin. The BF identified a resin cement-dentin diffusion zone of $1.8 \pm 0.4 \mu \mathrm{m}$ for the self-adhesive cement and $2.5 \pm 0.3 \mu \mathrm{m}$ for the self-etch cement. This analysis allowed the authors to estimate the diffusion of the resin cements into the dentin. Fitting the MRS data to the BF contributed to and is relevant for future studies of the adhesive interface. $\odot$ The Authors. Published by SPIE under a Creative Commons Attribution 3.0 Unported License. Distribution or reproduction of this work in whole or in part requires full attribution of the original publication, including its DOI. [DOI: 10.1117/1.JBO.21.5.055003]
\end{abstract}

Keywords: resin cements; dentin; adhesion.

Paper 150794RR received Dec. 2, 2015; accepted for publication Apr. 27, 2016; published online May 27, 2016.

\section{Introduction}

Adhesion to tooth substrates is fundamentally based on a process whereby the mineral from the superficial region is partially removed and replaced by polymerized resin monomers. ${ }^{1}$ This process, known as micromechanical interlocking or hybridization, is believed to induce hybrid layer formation at the adhesive interface. ${ }^{2}$ Establishment of the hybrid layer has been considered important for the bond formed between adhesive materials and the tooth, a significant factor in achieving optimal long-term dental restorations. ${ }^{3}$

Traditionally, the hybrid layer formed at the interface between adhesive cements and the dentin has been assessed by scanning electron microscopy (SEM), transmission electron microscopy (TEM), or light microscopy. ${ }^{4}$ These morphological imaging techniques have provided substantial insight into the morphological characteristics of resin cement diffusion into dentin that consists of a specific region at the interface, where resin monomers penetrate into the smear layer and demineralized dental hard tissues. ${ }^{5}$ Features such as the extent and uniformity of resin penetration into dentin; appearance; length and continuity of resin tags; quality of hybridization; and hybrid layer thickness have been assessed. ${ }^{6-9}$ Although micromechanical retention resulting from the presence of a hybrid layer and resin tags has been considered the main adhesion mechanism between adhesives and dental substrates, the potential benefit of

*Address all correspondence to: Renata Corrêa Pascotto, E-mail: renatapascotto@gmail.com additional chemical interactions between functional monomers and the tooth has recently gained attention. ${ }^{1,10}$

Recently, self-adhesive resin cements have been introduced to the market. Different from the traditional etch-and-rinse or selfetch adhesive materials, self-adhesive cements do not require pretreatment of the tooth surface, and they are applied in a single clinical step, similar to the use of conventional zinc-phosphate or glass ionomer cements. ${ }^{11}$ Self-adhesive resin cement bonds are attributed to the reaction of acidic monomers present in their formulation with the hydroxyapatite of the dental structure, resulting in chemical and micromechanical retention. ${ }^{12}$ However, several studies based on different morphological imaging methods have been unable to demonstrate evidence of dentin decalcification achieved by the self-adhesive resin cements investigated and their infiltration into dentin. ${ }^{13-16}$ Although studies have observed an intimate adaptation of self-adhesive resin cements to dentin, no hybrid layer or resin tag formation could be identified. ${ }^{17-19}$ An established hybridization is typically observed with the use of total-etch or self-etch cements, whereas more superficial physical interactions and possible involvement of chemical reactions between the material and dental hard tissues have been found with self-adhesive cements. ${ }^{13}$

Micromorphological analyses of adhesive interfaces, performed either between the tooth and adhesive systems ${ }^{20-22}$ or between the tooth and resin cements, ${ }^{23,24}$ have made important contributions to the correct use of adhesive materials, with the aim of improved bonding efficiency and durability. More recently, micro-Raman spectroscopy (MRS) has been used as an alternative method to the traditional morphological imaging 
techniques for examining the adhesive interface. ${ }^{6,25-28}$ This method uses a laser beam to scan the area of interest. MRS enables spectra to be collected of the different components present at the adhesive interface. Furthermore, this method identifies the Raman bands associated with the chemical bonds (functional groups), thus allowing researchers to distinguish different materials more precisely. The different Raman bands work like fingerprints for each material analyzed. By observing the different intensities of the spectral bands, variations in the resin cementdentin diffusion zones between the adhesive materials and dental substrates can be established. ${ }^{29,30}$ Thus, MRS may be an important tool to gain better understanding of the nature of the interface between adhesive cements and dentin.

The purposes of the present study were to analyze the interfaces between two resin luting agents and dentin by means of MRS and to present a mathematical model for estimating the diffusion zone of resin cements into dentin. It was hypothesized that this analysis would allow the authors to measure the diffusion into dentin of both self-etch and self-adhesive resin cements.

\section{Materials and Methods}

The study protocol was approved by the Local Ethics Committee (CAAE No. 19425213.2.0000.0104). Nine noncarious human third molars were used in the study $(n=9)$.

\subsection{Tooth Preparation and Cementation of Indirect Restorations}

Figure 1 illustrates sample preparation and analysis. The teeth were stored in saline solution at $4^{\circ} \mathrm{C}$ for no longer than six months. Class V cavities (height $3 \mathrm{~mm}$, length $5 \mathrm{~mm}$, depth $2 \mathrm{~mm}$ ) were prepared at the dentin-enamel junction on the buccal and lingual surfaces of the crown, by using high-speed diamond burs (No. 3131 KG Sorensen, Cotia, SP, Brazil) under water cooling. Due to the bur format selected, the cavities prepared had divergent and expulsive walls. In order to standardize the cavities, stickers with a window measuring $5 \mathrm{~mm}$ by $3 \mathrm{~mm}$ were placed on the tooth surface, and a rubber stopper was attached to the diamond bur in order to limit the preparation depth. A new diamond bur was used for each tooth. Class V cavity preparations made it possible to evaluate cement adhesion

\section{Tooth preparation and luting procedures}

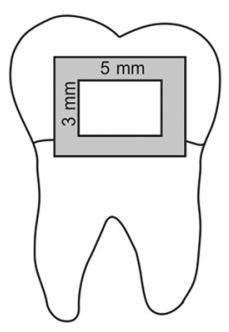

Tooth section

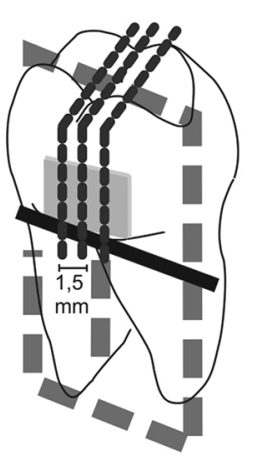

$1^{\circ}$ : bucco-lingual section

- 2०: mesio-distal section

$3^{\circ}$ : bucco-lingual section

Indirect restorations
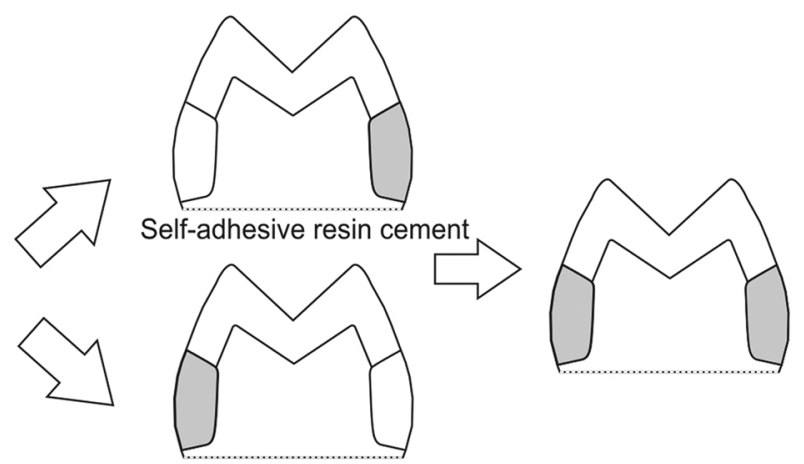

Self-etch resin cement
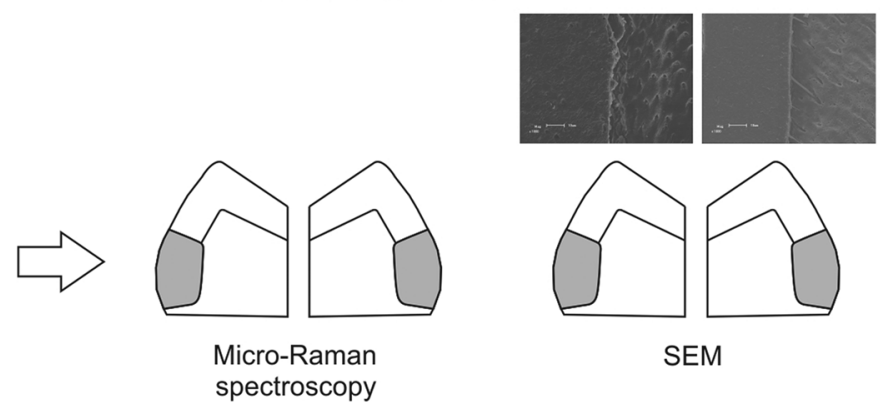

spectroscopy

\section{Micro-Raman spectroscopy}
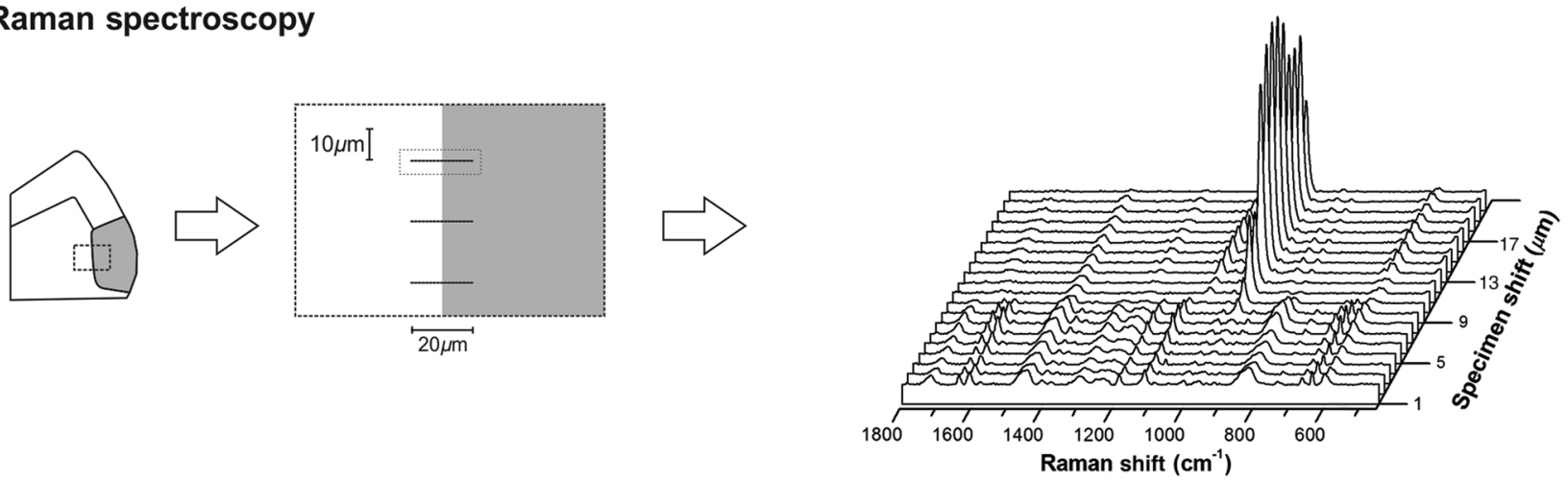

Fig. 1 Schematic drawing illustrating the study setup and specimen preparation. 
with axial wall dentin, avoiding extensive wear and allowing paired comparison analysis, by testing different cements in the buccal and lingual surface of the same tooth.

Inlay restorations were fabricated with composite resin (Filtek Z250 ${ }^{\circledR} 3 \mathrm{M}$ ESPE, St Paul, Minnesota). The cavities were lubricated with glycerin gel (Insulating gel, IvoclarVivadent, Schaan, Liechtenstein); resin composite was inserted in the cavity and light polymerized with a blue light-emitting diode (LED) light source of $1200 \mathrm{~mW} / \mathrm{cm}^{2}$ for $20 \mathrm{~s}$. The cavities were rinsed with water and air dried, both for $5 \mathrm{~s}$, before cementation.

Luting procedures were performed in a warm room at $37^{\circ} \mathrm{C} .{ }^{20}$ The inlays were luted either with a self-adhesive resin cement (RelyXUnicem $2^{\circledR}$ Clicker, 3M ESPE) or a self-etch resin cement (Multilink ${ }^{\circledR}$ Automix, Ivoclar-Vivadent). The luting agent used on the buccal or lingual surface was randomly chosen by draw. In order to allow direct comparisons, both cements were tested in a paired way, i.e., the same tooth received both luting agents. The luting procedures are described in detail in Table 1 . The resin cements were light polymerized using the same LED device used to light polymerize the composite inlays.

\subsection{Micro-Raman Spectroscopy}

After cementation, the specimens were stored in distilled water for $24 \mathrm{~h}$. Then the teeth were fixed with sticky wax and sectioned with a diamond saw (South Bay Technology Inc.,
California) adapted to a low-speed cutting machine (Isomet 1000, Buehler, Lake Bluff, Illinois) under water cooling. Since each tooth had the two analyzed material specimens, the cuts were made in the bucco-lingual direction from the occlusal to the cervical area, across both resin cement-dentin interfaces at the same time, resulting in two slabs $1.5 \mathrm{~mm}$ thick (Fig. 1). The inlays were divided into halves, one of which was measured by MRS technique and the other through SEM analysis. To avoid interface sample casting, the specimens were not metallographically polished; to minimize possible debris smeared across the specimen surface, they were submitted to an ultrasonic bath with distilled water for $5 \mathrm{~min}$.

Raman spectra were collected from the interface located at the axial wall of the cavities. The measurements were performed at room temperature with a SENTERRA dispersive Raman microscope (BrukerOptik GmbH, Ettlingen, Germany). The Raman spectra were measured using a micro-Raman system excited by a $785 \mathrm{~nm}$ laser source and registered at the spectral range of 450 to $1800 \mathrm{~cm}^{-1}$. Laser power was set at $100 \mathrm{~mW}$ and focused on the sample through a $100 \times$ objective optical microscope $(0.75$ N.A.), using the $50 \mu \mathrm{m}$ confocal hole. The spectral resolution was $\sim 3$ to $5 \mathrm{~cm}^{-1}$; detector integration time was $3 \mathrm{~s}$; and the spectrum collected at each point was the average result of 60 consecutive readings. In order to improve signal-to-noise ratio, detector temperature was decreased to $-90^{\circ} \mathrm{C}$. All spectra were systematically collected under the same conditions. The area chosen for analysis in the first specimen was photographed

Table 1 Application mode of the resin cements tested.

Group Application mode Composition

RelyXUnicem $2^{\circledR} \quad$ Tooth cavity: no procedure

Clicker

Self-adhesive

Inlay restoration: no procedure

Luting procedure: application of the self-adhesive cement directly on the inlay; placement of the inlay into the cavity under manual pressure for $5 \mathrm{~s}$; removal of excess cement; light activation for $20 \mathrm{~s}$, application of glycerin gel; light activation for additional $20 \mathrm{~s}$.
Glass powder surface modified acrylic acid methacrylic acid Silane

bulk material

2-propenoic acid, 2-methyl-, 1,1'-[1- (hydroxymethyl)-1,2-ethanediyl] ester,

reaction products with 2-hydroxy-1,3- propanediyl dimethacrylate and

phosphorus oxide

TEDGDMA

Silane treated silica

Sodium persulfate

Oxide glass chemicals (nonfibrous)

Tert-butyl peroxy-3,5,5- trimethylhexanoate

Cement: dimethacrylate, HEMA, barium glass, ytterbium trifluoride, and spheroid mixed oxide

Primer A: aqueous solution of initiators

Primer B: HEMA, phosphonic acid, and methacrylate monomers
Cementing: application of the self-etch cement on the inlay; placement of the inlay into the cavity under manual pressure for $5 \mathrm{~s}$; removal of excess cement; light activation for $20 \mathrm{~s}$, application of glycerin gel; light activation for further $20 \mathrm{~s}$. 
and served as a reference for locating the interface in the subsequent specimens. Spectra were acquired at positions corresponding to $1-\mu \mathrm{m}$ intervals across the interface between the resin cement and dentin ${ }^{31-38}$ by using the computer controlled $x-y-z$ stage with a minimum step width of $50 \mathrm{~nm}$. Raman spectra were collected along three line-scans for each slab, starting in the dentin and ending in the cement resin layer, each $20 \mu \mathrm{m}$ long, with $10 \mu \mathrm{m}$ space between lines. To avoid specimen dehydration, gauze soaked in distilled water was placed in contact with the dentin to keep it moist during MRS measurement.

\subsection{Analysis of the Resin Cements-Dentin Diffusion Zone}

The resin cement-dentin diffusion zone between the resin cements and dentin was analyzed by a computer program for interactive scientific graphing and data analysis (Origin, Northampton, Massachusetts). Band intensity peaks at $961 \mathrm{~cm}^{-1}(\nu 1$ phosphate symmetric stretch), representative of the phosphate present in dentin, and at $\sim 1113 \mathrm{~cm}^{-1}(\mathrm{C}-\mathrm{O}-\mathrm{C})$, representative of the carbon chain in the resin monomer (present in both cements), were used to identify the resin cement-dentin diffusion zones. Peaks located at $\sim 1113 \mathrm{~cm}^{-1}$ have previously been used as reference for identifying resin monomers. ${ }^{29,30}$ Nonetheless, in the present study, each spectrum was individually verified to ensure that this peak was present only in the cements tested. The evolution of peak intensities of the Raman bands collected from the functional groups corresponding to the resin monomer present in the cements was sigmoid shaped functions.

A sigmoid function is usually used to determine the rate of growth, starting from low values, passing through its maximum at a point of inflexion, and then reaching a saturation value. The curve looks like a rotated $\mathrm{S} .{ }^{39}$ The inflexion point may be associated with a transition region from the initial to the final stage of the system investigated (Fig. 2). In this study, the experimental data fitted an S-shaped curve, best described by the Boltzmann function. ${ }^{40}$

Figure 2 is representative of the Boltzmann function, which is described by $f(x)=\left(\left(A_{1}-A_{2}\right) /\left\{1+\exp \left[\left(x-x_{0}\right) / d x\right]\right\}\right)+A_{2}$, where $A_{1}$ and $A_{2}$ are the initial and final plateau of the peak band intensity at $1113 \mathrm{~cm}^{-1}$, which corresponded to the dentin and

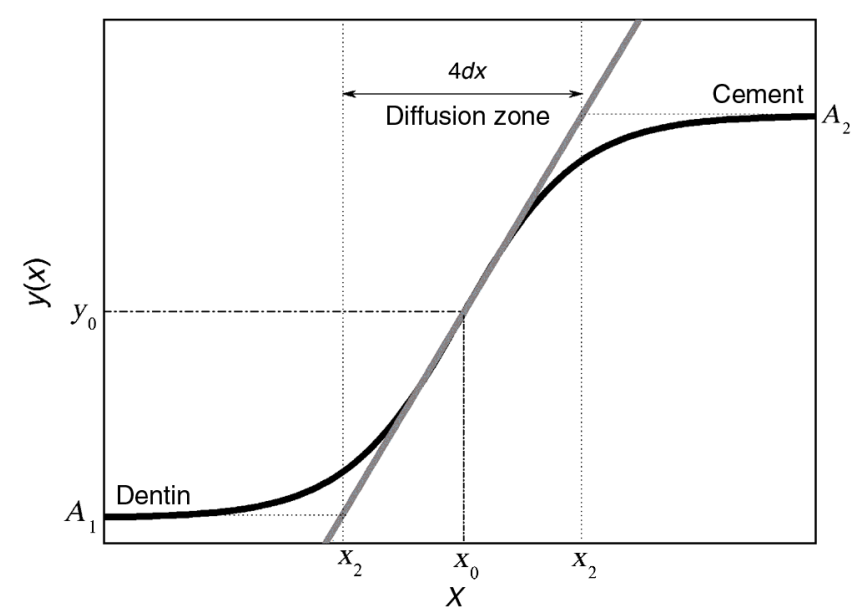

Fig. 2 Boltzmann function used in this study to fit the experimental MRS data; $A_{1}$ and $A_{2}$, the initial and final mean values for the dentin and resin cement band intensities; $4 d x$, the resin cements-dentin diffusion zone width. cement region of the sample, respectively. $x$ is the displacement across the interface formed by the adhesive cement and dentin, while $x_{0}$ represents the midpoint of the resin cement-dentin diffusion zone. The $y$ axis is the Raman signal intensity. The diffusion zone is the region delimited by a straight-line tangent to the midpoint $\left(x_{0}, y_{0}\right)$. The straight tangent line is mathematically described by a linear equation, i.e., $g(x)=a x+b$, where $a$ and $b$ are constants. The constant $=\left[\left(A_{2}-A_{1}\right) / 4 d x\right]$ is obtained by the first derivate of the Boltzmann function at $x=x_{0}$, and the constant $=\left[\left(A_{2}+A_{1}\right) / 2\right]-\left\{\left[\left(A_{2}-A_{1}\right) / 4 d x\right] x_{0}\right\}$ is obtained by replacing the values $x=x_{0}$ and $y=y_{0}=\left[\left(A_{2}+A_{1}\right) / 2\right]$ in the $g(x)$ linear function. By replacing $a$ and $b$ values in $g(x)$, it is possible to obtain the distance between $x_{0}$ and the two intersection points $x_{1}$ and $x_{2}$ that correspond to $4 d x$, which represents the width of the resin cement-dentin diffusion zones. Thus, by fitting the experimental Raman data collected from the scanned lines to the Boltzmann function, the authors could acquire the $d x$ parameter and determine the width of the resin cement-dentin diffusion zone.

The sigmoidal fitting was performed only for the data collected from the intensity of characteristic peaks associated with cement $\left(1113 \mathrm{~cm}^{-1}\right)$, since the resin cements permeated into the dentin. The characteristic peak associated with dentin $\left(961 \mathrm{~cm}^{-1}\right)$ that represents the mineral component of the tooth did not have a constant distribution across the measurement and was therefore used only as a reference of cement monomer diffusion and was not fitted to the function.

The thickness data were checked for normality using the Shapiro-Wilk test and analyzed by Student's $t$ test for the cement adhesive strategy factor (R-statistics software). Statistical significance was set at $p<0.05$.

In addition, chemical and physical changes in the spectral region between the dentin and cement were investigated by visual analyses of Raman bands at each individual spectrum for the two groups.

\subsection{Scanning Electron Microscopy Analysis}

The three specimens selected for SEM (SS-550 Superscan, Shimadzu Biotech, Japan) were dehydrated in increasing series of ethanol for $15 \mathrm{~min}$ in each solution of 80 and $90 \%$, and for $30 \mathrm{~min}$ in absolute ethanol. The specimens were then sputtercoated with a layer of gold, and photomicrographs (1000× magnification) were taken for visual analysis of the cement/dentin interface.

\section{Results}

From the MRS spectra, the authors identified the resin cementdentin diffusion zones between the dentin and the resin cements. The characteristic peaks associated with dentin and cement peaks were simultaneously detected for both the self-adhesive [Fig. 3(a)] and the self-etch [Fig. 3(b)] cements. Boltzmann analysis showed significantly lower values $(p<0.05)$ for the resin cement-dentin diffusion zones of self-adhesive cement $(1.8 \pm 0.4 \mu \mathrm{m})$ when compared to self-etch cement $(2.5 \pm 0.3 \mu \mathrm{m})$ [Figs. 4(a) and 4(b)]. Visual analyses of the individual spectra showed that the Raman band correspondent to $600 \mathrm{~cm}^{-1}$ ( $\nu 4$ phosphate bending mode) was modified in the spectral region between the dentin and cement, thus suggesting the interaction of these materials and representing the resin cement-dentin diffusion zones (Fig. 5).

Scanning electron photomicrography analysis showed differences in the interface between the self-adhesive or 


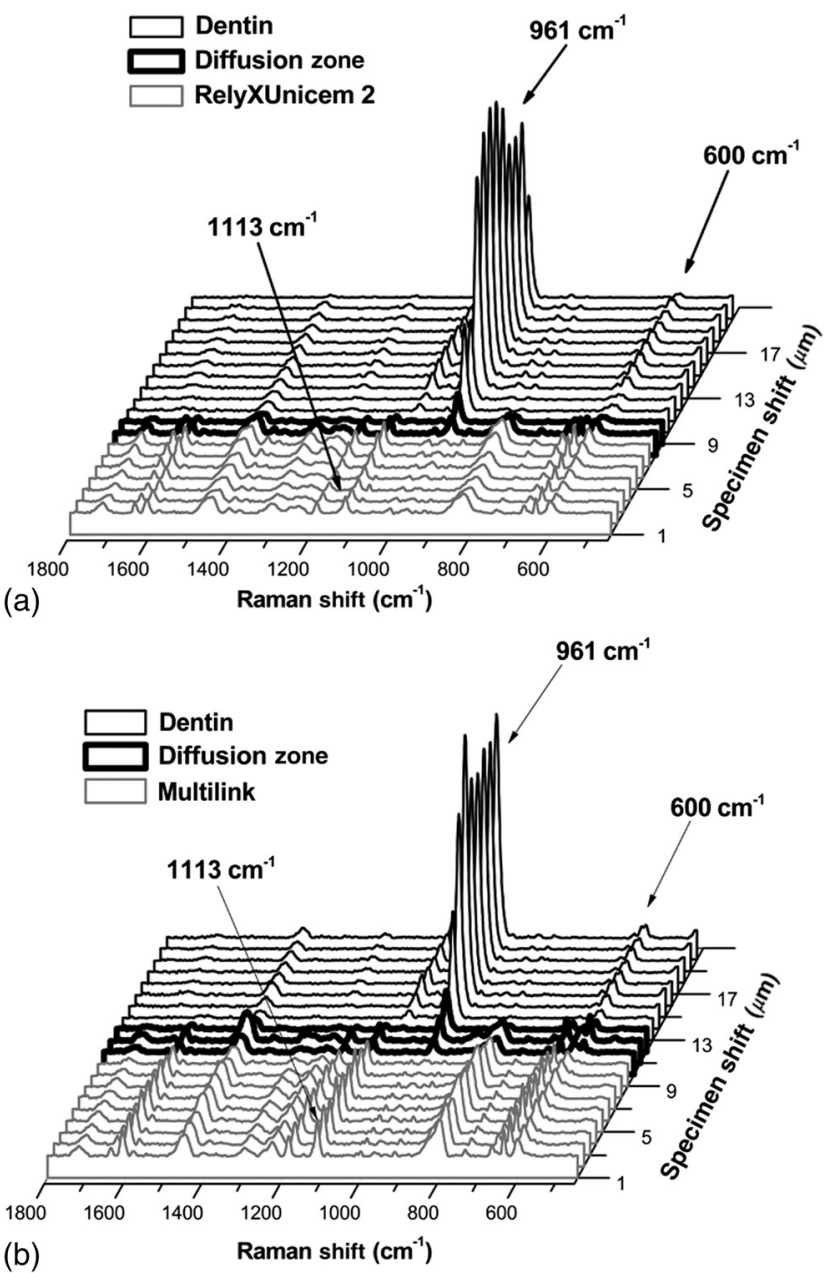

Fig. 3 Representative Raman spectra of lines measured across the dentin-cement interface. The shift scale is represented by a numbered line: (a) RelyXUnicem $2^{\circledR}$ and (b) Multilink ${ }^{\circledR}$.

self-etching cements and the dentin. Self-adhesive cement presented an interface that was indistinguishable from dentin [Fig. 6(a)], while the self-etching cement presented a clear transition line with long projections of the material (tags) inside the demineralized dentin [Fig. 6(b)].

\section{Discussion}

To the best of our knowledge, this is the first study to propose a mathematical model based on Raman spectral readings to estimate the diffusion zone at the interface between resin cements and dentin. The results of the present study demonstrated that there are differences in the thickness of the transition layer between dentin/self-etching cement and dentin/self-adhesive cement. These results were confirmed by the analysis of the photomicrographs that showed that the projections of the selfetching cement were deeper in the dentin, while the tags of the self-adhesive cement showed small penetration (extension) through the dentin. The sigmoidal fitting of the S-shaped curve produced by the MRS data (Fig. 4) resulting from the application of the Boltzmann function to the intensity of the band at $1113 \mathrm{~cm}^{-1}$ allowed the authors to estimate the thickness of the resin cement-dentin diffusion zones. With this new tool, important insight was obtained into the cements investigated.

To date, most studies assessing the interface between adhesive materials and dentin have used SEM, TEM, or light
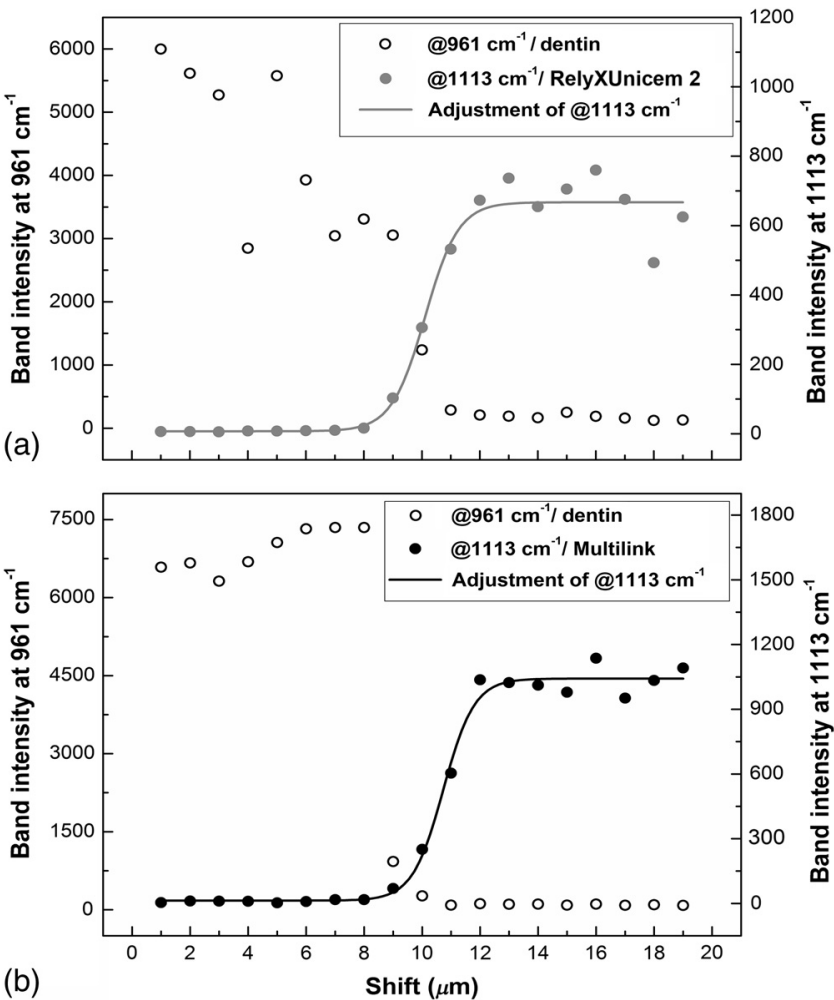

Fig. 4 Ratio of the Raman spectra at $961 \mathrm{~cm}^{-1}(\nu \mathrm{P}-\mathrm{O}$, dentin) to $1113 \mathrm{~cm}^{-1}$ ( $\nu \mathrm{C}-\mathrm{O}-\mathrm{C}$, cement) of one measurement line, and fit to the Boltzmann function at $1113 \mathrm{~cm}^{-1}$. The black spots represent the plot of dentin peaks, which were used only to verify the behavior of dentin in the scanned sample: (a) dentin/RelyXUnicem $2{ }^{\circledR}$ diffusion zone and (b) dentin/Multilink ${ }^{\circledR}$ diffusion zone.

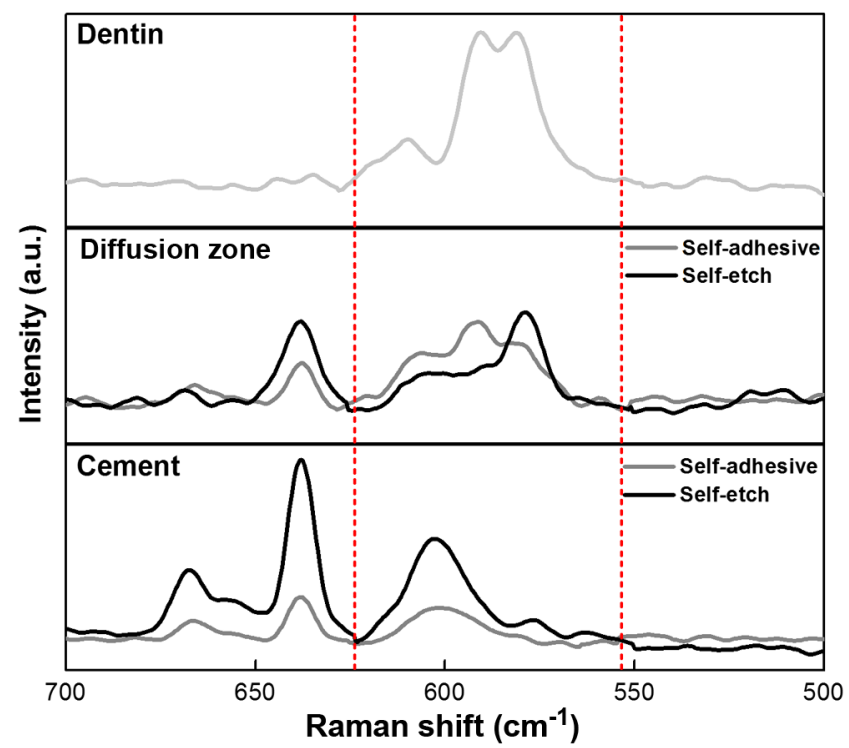

Fig. 5 Illustration of the Raman spectra of a single phosphate band of dentin, the resin cement-dentin diffusion zone, and the resin cements. The spectra delimitation between 627 and $550 \mathrm{~cm}^{-1}$ showed the peak centered at $600 \mathrm{~cm}^{-1}$, demonstrating that at the resin cement-dentin diffusion zone spectra, the phosphate band pattern was altered. Gray line: dentin; dark gray line: RelyXUnicem $2^{\circledR}$; black line: Multilink ${ }^{\circledR}$. 


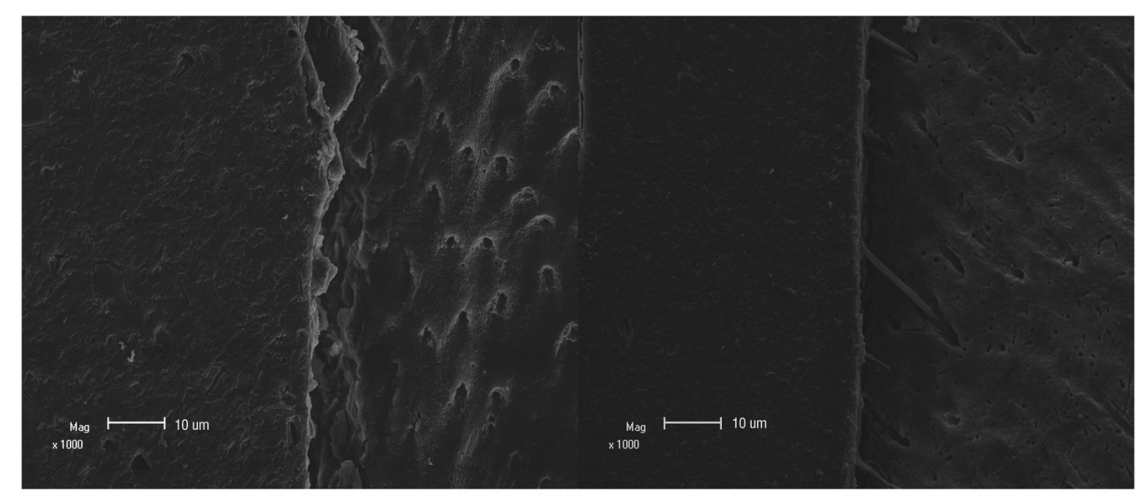

(a)

(b)

Fig. 6 Representative scanning electron micrographs of the adhesive/cement interface: (a) RelyXUnicem $2^{\circledR}$ and (b) Multilink ${ }^{\circledR}$

microscopy. Visual analysis of the adhesive interface using these methods can be influenced by the quality of the area being analyzed on the photomicrograph and by sample preparation (sectioning position; level of demineralization; and exposure of dentin tubules). These factors most probably contributed to the variability of the results seen among earlier studies. ${ }^{5,12,13,18}$

In the search for a more objective procedure to analyze the adhesive interface, Zhang and Wang ${ }^{30}$ used MRS. Based on the presence of bands representative of dentin and adhesives simultaneously in the same spectrum, the authors were able to locate the adhesive-dentin interface and to estimate the effect of different application modes of the adhesives on hybrid layer formation. In the present study, in a similar manner, a region simultaneously containing traces of $\mathrm{P}-\mathrm{O}$ (dentin) and $\mathrm{C}-\mathrm{O}-\mathrm{C}$ (resin monomer present in the cements) was detected at the cement-dentin interface for both self-etch and self-adhesive cements.

The results from the present study partly exceed those of previous studies. While morphological imaging techniques have demonstrated a thin hybrid layer for self-etch cements, no such formation was observed for self-adhesive cements, despite the intimate adaptation between the cement and the dental substrate. ${ }^{13-19}$ De Munck et al., ${ }^{19}$ with the use of TEM analysis, observed an irregular interaction zone at the interface between dentin and a self-adhesive cement, ranging from 0 up to $2 \mu \mathrm{m}$.

When luting with self-adhesive cements, the dentin tubules were not shown to be infiltrated by the cement. ${ }^{5}$ Moreover, selfadhesive cements were not able to completely dissolve the smear layer. ${ }^{5,19}$ In the present study, we observed an area in which the bands representative of dentin and the self-adhesive cement were simultaneously found in the same spectrum. This clearly demonstrated resin cement diffusion into dentin. A possible explanation is that the smear layer resulting from cavity preparation with diamond burs, usually 1 to $2 \mu \mathrm{m}$ thick, ${ }^{41,42}$ was infiltrated by resin monomers ${ }^{19,43}$ due to pressure during luting. 5,19,44 Thus, the MRS results for the self-adhesive cement identified the phosphate present in the smear layer combined with the resin monomer present in the cement.

Mild self-etch adhesives ( $\mathrm{pH} \sim 2$ ) have been reported to form a thin hybrid layer of $\sim 1 \mu \mathrm{m}$, while an interaction between 1 and $2 \mu \mathrm{m}$ has been observed with strong $(\mathrm{pH}<1)$ self-etch adhesives. ${ }^{1}$ The smear layer formed by this type of adhesive is never rinsed away, but partly dissolved and incorporated into the hybrid layer. In the present study, the slightly higher value found for the diffusion zone of the mild self-etch cement tested $(2.4 \pm 0.4 \mu \mathrm{m})$ may be explained by the fact that the mineral content present in the dentin and in the smear layer contributed to the measurement of the resin cement diffusion into dentin. Therefore, only part of this value actually refers to the presence of a true hybrid layer.

The results from this study open the possibility for future research, such as assessing the adhesive interface with MRS on smear layer-free dentin surfaces (e.g., fractured dentin) to elucidate this matter. MRS ensures spectral readings that are extremely precise and representative of the materials in the area analyzed. This method does not require surface preparation such as those needed for the morphological imaging techniques, thereby minimizing the possibility of artifacts. However, readings must be verified for possible discrepancies due to the presence of air included at the interface. The main problem with the use of MRS, however, concerns its limited spatial resolution. When 100× objective lenses are used, the laser spot is $\sim 1 \mu \mathrm{m}$, while the adhesive interface may be $<1 \mu \mathrm{m}$, as shown by ultramorphological interfacial analysis using TEM. ${ }^{5}$ Since MRS analysis contained hundreds of very high-quality spectra at a spatial resolution of $1 \mu \mathrm{m},{ }^{31-38}$ the difference in phosphate and monomer composition can be determined across the length and thickness of the resin cement diffusion into dentin, ${ }^{25,29}$ with the advantage of the fingerprint characteristics of the Raman spectral bands.

Lenses with $100 \times$ magnification intensify irregularities in the resin cement-dentin diffusion zones of resin monomers. To overcome this inert feature of the samples, three linear scans were performed on each sample in order to characterize the interface at different positions along the sample. Although the position of the resin cement-dentin diffusion zones differed from one line to another due to irregularities in this region, the chemical analysis with MRS provided a comprehensive representation of the thickness of the resin cement-dentin diffusion zones at the interface. This representation demonstrated a regular pattern for both the self-adhesive $(1.8 \pm 0.4 \mu \mathrm{m})$ and mild self-etch cement $(2.5 \pm 0.3 \mu \mathrm{m})$. The Raman bands obtained with this research are considered fingerprints of specific molecules contained in the specimens. As a result, they provided information on the chemical interactions, expressed by alterations and/or appearance of new peaks. ${ }^{30}$ The MRS technique enabled the evaluation of the diffusion zone chemical compounds and consequently measured its dimension from its structural composition, ${ }^{31-38}$ while in imaging techniques, the interface is estimated in a qualitative approach. ${ }^{6,25-28}$ Therefore, due to the difference of the characteristics analyzed with these techniques, it is not appropriate to validate 
the analytical method proposed in this research through imaging techniques.

In conclusion, MRS was shown to be a simple and reliable method to assess the resin cement diffusion into dentin. The S-shaped curve fitted by the application of the Boltzmann function allowed the authors to estimate the resin cement-dentin diffusion zones. This analysis supplied important information on the extent and nature of the resin cement-dentin diffusion zones. It may benefit future adhesive interface studies of both self-etch and self-adhesive resin cements.

\section{Acknowledgments}

The authors thank the Brazilian funding agencies FINEP (Financiadora de Estudos e Projetos) and CAPES (Coordenação de Aperfeiçoamento de Pessoal de Nível Superior) for their financial support. All the authors declare no potential conflicts of interest with respect to the authorship and/or publication of this article.

\section{References}

1. B. Van Meerbeek et al., "State of the art of self-etch adhesives," Dent. Mater. 27, 17-28 (2011).

2. N. Nakabayashi, K. Kojima, and E. Masuhara, "The promotion of adhesion by the infiltration of monomers into tooth substrates," J. Biomed. Mater. Res. 16, 265-273 (1982).

3. G. Inoue et al., "Morphological categorization of acid-base resistant zones with self-etch primer adhesive systems," Dent. Mater. J. 31, 232-238 (2012).

4. J. A. Skupien et al., "Micromorphological effects and the thickness of the hybrid layer-a comparison of current adhesive systems," J. Adhes. Dent. 12, 435-442 (2010).

5. F. Monticelli et al., "Limited decalcification/diffusion of self-adhesive cements into dentin," J. Dent. Res. 87, 974-979 (2008).

6. B. Van Meerbeek et al., "Comparative SEM and TEM examination of the ultrastructure of the resin-dentin interdiffusion zone," J. Dent. Res. 72, 495-501 (1993).

7. L. Breschi et al., "Morphological study of resin-dentin bonding with TEM and in-lens FESEM," Am. J. Dent. 16(4), 267-274 (2003).

8. B. Van Meerbeek et al., "Correlative transmission electron microscopy examination of nondemineralized and demineralized resin-dentin interfaces formed by two dentin adhesive systems," J. Dent. Res. 75, 879-888 (1996).

9. Y. Wang and P. Spencer, "Effect of acid etch time and technique on interfacial characteristics of adhesive-dentin bond using differential staining," Eur. J. Oral Sci. 112, 293-299 (2004).

10. Y. Yoshida et al., "Comparative study on adhesive performance of functional monomers," J. Dent. Res. 83(6), 454-458 (2004).

11. A. M. Diaz-Arnold, M. A. Vargas, and D. R. Haselton, "Current status of luting agents for fixed prosthodontics," J. Prothet. Dent. 81(2), 135141 (1999).

12. K. Hikita et al., "Bonding effectiveness of adhesive luting agents to enamel and dentin," Dent. Mater. 23(1), 71-80 (2007).

13. T. R. Aguiar et al., "Micromorphology of resin-dentin interfaces using selfadhesive and conventional resin cements: a confocal laser and scanning electron microscope analysis," Int. J. Adhes. Adhes. 38, 69-74 (2012).

14. T. R. Aguiar et al., "Interfacial ultramorphology evaluation of resin luting cements to dentin: a correlative scanning electron microscopy and transmission electron microscopy analysis," Microsc. Res. Tech. 76, 1234-1239 (2013).

15. K. Al-Assaf et al., "Interfacial characteristics of adhesive luting resins and composites with dentine," Dent. Mater. 23, 829-839 (2007).

16. K. Bitter et al., "Reliability of fiber post bonding to root canal dentin after simulated clinical function in vitro," Oper. Dent. 37, 397-405 (2012).

17. I. Radovic et al., "Self-adhesive resin cements: a literature review," J. Adhes. Dent. 10(4), 251-258 (2008).

18. R. R. Vaz et al., "Bond strength and interfacial micromorphology of etch-and-rinse and self-adhesive resin cements to dentin," $J$. Prosthodont. 21, 101-111 (2012).
19. J. De Munck et al., "Bonding of an auto-adhesive luting material to enamel and dentin," Dent. Mater. 20, 963-971 (2004).

20. M. T. De Oliveira et al., "Micromorphology of resin-dentin interfaces using one-bottle etch\&rinse and self-etch adhesive systems on lasertreated dentin surfaces: a confocal laser scanning microscope analysis," Lasers Surg. Med. 42, 662-670 (2010).

21. T. Pioch, "Applications of confocal laser scanning microscopy to dental bonding," Adv. Dent. Res. 11, 453-461 (1997).

22. F. Reis et al., "Interfacial ultramorphology of single-step adhesives: nanoleakage as a function of time," J. Oral Rehabil. 34, 213-221 (2007).

23. C. A. Arrais et al., "Micromorphology of resin/dentin interfaces using 4th and 5th generation dual-curing adhesive/cement systems: a confocal laser scanning microscope analysis," J. Adhes. Dent. 11(1), 15-26 (2009).

24. K. Bitter et al., "Correlation of scanning electron and confocal laser scanning microscopic analyses for visualization of dentin/adhesive interfaces in the root canal," J. Adhes. Dent. 11(1), 7-14 (2009).

25. P. Spencer et al., "Interfacial chemistry of the dentin/adhesive bond," J. Dent. Res. 79(7), 1458-1463 (2000).

26. R. M. Lemor et al., "Spectroscopic and morphologic characterization of the dentin/ adhesive interface," J. Biomed. Opt. 4(1), 22-27 (1999).

27. M. Ozaki et al., "Laser-Raman spectroscopic study of the adhesive interface; analysis between 4-META/MMA-TBB resin and bovine or human dentin," Dent. Mater. J. 11(1), 70-76 (1992).

28. M. Suzuki, H. Kato, and S. Wakumoto, "Vibrational analysis by Raman spectroscopy of the interface between dental adhesive resin and dentin," J. Dent. Res. 70(7), 1092-1097 (1991).

29. T. P. Shin et al., "Morphological and chemical characterization of bonding hydrophobic adhesive to dentin using ethanol wet bonding technique," Dent. Mater. 25, 1050-1057 (2009).

30. Y. Zhang and Y. Wang, "Effect of application mode on interfacial morphology and chemistry between dentine and self-etch adhesives," J. Dent. 41, 231-240 (2013).

31. B. van Meerbeek et al., "Chemical characterization of the resin-dentin interface by micro-Raman spectroscopy," J. Dent. Res. 72, 1423-1428 (1993).

32. Y. Wang and P. Spencer, "Quantifying adhesive penetration in adhesive/ dentin interface using confocal Raman microspectroscopy," J. Biomed. Mater. Res. 59(1), 46-55 (2002).

33. R. Parthasarathy et al., "Application of multivariate spectral analyses in micro-Raman imaging to unveil structural/chemical features of the adhesive/dentin interface," J. Biomed. Opt. 13(1), 014020 (2008).

34. Y. Wang et al., "Effect of solvent content on resin hybridization in wet dentin bonding," J. Biomed. Mater. Res. A 82(4), 975-983 (2007).

35. X. Guo et al., "Effects of a solubility enhancer on penetration of hydrophobic component in model adhesives into wet demineralized dentin," Dent. Mater. 23(12), 1473-1481 (2007).

36. Y. Wang, P. Spencer, and M. P. Walker, "Chemical profile of adhesive/ caries-affected dentin interfaces using Raman microspectroscopy," J. Biomed. Mater. Res. A 81(2), 279-286 (2007).

37. Y. Wang, P. Spencer, and X. Yao, "Micro-Raman imaging analysis of monomer/mineral distribution in intertubular region of adhesive/dentin interfaces," J. Biomed. Opt. 11(2), 024005 (2006).

38. P. Spencer, Y. Wang, and B. Bohaty, "Interfacial chemistry of moistureaged class II composite restorations," J. Biomed. Mater. Res. B Appl. Biomater. 77(2), 234-240 (2006).

39. P. D. C. Birch, "A new generalized logistic sigmoid growth equation compared with the Richards growth equation," Ann. Bot. 83, 713-723 (1999).

40. OriginLab Corporation, "Origin help," 2016, http://www.originlab.com/ doc/Origin-Help/Boltzmann-FitFunc (16 May 2016).

41. D. H. Pashley et al., "Scanning electron microscopy of the substructure of smear layers in human dentine," Arch. Oral Biol. 33, 265-270 (1988).

42. R. B. Ermis et al., "Bond strength of self-etch adhesives to dentin prepared with three different diamond burs," Dent. Mater. 24, 978-985 (2008).

43. F. R. Tay et al., "Effect of smear layers on the bonding of a self-etching primer to dentin," J. Adhes. Dent. 2(2), 99-116 (2000).

44. C. Goracci et al., "Microtensile bond strength and interfacial properties of self-etching and self-adhesive resin cements used to lute composite onlays under different seating forces," J. Adhes. Dent. 8(5), 327-335 (2006).

Biographies for the other authors are not available. 